(RESEARCH ARTICLE)

\title{
Effectiveness of selected broad spectrum antibiotics against Pseudomonas species isolated through the use of chloramphenicol
}

\author{
Peekate Lekiah Pedro * and Biidee Barididum \\ Department of Microbiology, Faculty of Science, Rivers State University, P. M. B. 5080 Port Harcourt, Nigeria.
}

Publication history: Received on 08 December 2018; revised on 20 December 2018; accepted on 24 December 2018

Article DOI: https://doi.org/10.30574/gscbps.2018.5.3.0158

\begin{abstract}
Greenish pigment producing Pseudomonas species were isolated from water samples using nutrient agar supplemented with $50 \mu \mathrm{g} . \mathrm{ml}^{-1}$ chloramphenicol. Broth cultures of three of the Pseudomonas isolates were prepared for minimum inhibitory concentration (MIC) determination by adjusting their turbidity using sterile normal saline to match the turbidity of McFarland standard No. 1 which is regarded to have a cell density of about $3 \times 10^{8} \mathrm{cfu} / \mathrm{ml}$. The MIC of ciprofloxacin, erythromycin, and tetracycline against the three isolates was determined using the agar dilution method. The concentrations of the antibiotics used are $0.625,1.25,2.5,5.0,10.0,20.0,40.0,80.0,160.0,320.0$, and $640.0 \mu \mathrm{g} / \mathrm{ml}$. The results showed that the MIC of ciprofloxacin against the Pseudomonas isolates ranged from 5 - $640 \mu \mathrm{g} / \mathrm{ml}$; the MIC of tetracycline against the isolates ranged from $5-640 \mu \mathrm{g} / \mathrm{ml}$; and the MIC of erythromycin against the isolates ranged from $40-80 \mu \mathrm{g} / \mathrm{ml}$. It is thus concluded that multidrug resistant species of Pseudomonas can be generated as a result of using chloramphenicol for the isolation of Pseudomonas, and that erythromycin can be effective in treating infections caused by Pseudomonas species that are resistant to ciprofloxacin and tetracycline.
\end{abstract}

Keywords: Pseudomonas species; Chloramphenicol; Minimum inhibitory concentration; Broad spectrum antibiotics; Multi-drug resistance

\section{Introduction}

Cultures of Pseudomonas species are often required for research, environmental and industrial application. This is due to their ability to metabolize and transform several organic compounds, and also their ability to produce biosurfactants and other organic molecules [1-3]. Due to their potential importance and application, these organisms are often sought after by researchers and bio-industrialists. Isolation of Pseudomonas species from environmental media using nutrient agar supplemented with chloramphenicol has been suggested by Peekate and Abu [4]. Their suggestion was based on the observation that certain species of Pseudomonas produces greenish pigment during growth in the presence of chloramphenicol.

Chloramphenicol is a broad spectrum antibiotic used in the treatment of infections such as conjunctivitis, typhoid fever, and otitis media [5-8]. Been a broad spectrum antibiotic, it is expected to be effective in the treatment of infections caused by different bacterial groups. Chloramphenicol is bacteriostatic in nature [9]; it does not actually kill bacterial pathogens, but stops them from multiplying and carrying out certain metabolic activities. As a result of not been killed, some bacterial pathogens develop resistance to the antibiotic, especially when the antibiotic is applied at sub-inhibitory concentrations.

The use of chloramphenicol in the isolation of Pseudomonas, while suppressing the population of other bacteria, can lead to the development of multi-drug resistance in the Pseudomonas species isolated. Determining the potency of

\footnotetext{
${ }^{*}$ Corresponding author

E-mail address: lekia.peekate@ust.edu.ng
} 
selected broad spectrum antibiotics against bacteria isolated using chloramphenicol or other antibiotics is thus necessary so as to be prepared for laboratory acquired infections that may occur due to work with such bacteria. This will also make known the antibiotic of choice for treatment of infections that could arise as a result of escape of such bacteria from the laboratory.

Infections caused by multi-drug resistant species of Pseudomonas can be tackled through the use of broad spectrum antibiotics. Broad spectrum antibiotics, among other usefulness, are useful for treating infections caused by antibioticresistant bacteria [10]. Examples of broad spectrum antibiotics include amoxicillin, augmentin, imipenem, chloramphenicol, ciprofloxacin, erythromycin, and tetracycline $[9,11]$. Before the use of a broad spectrum antibiotic in the treatment of infection caused by an antibiotic-resistant bacterium, the effectiveness of the broad spectrum antibiotic against the resistant bacterium should be known so as to aid clinicians in making empirical decisions and prevent the further development and spread of multidrug resistant-bacteria. In investigating the effectiveness of an antimicrobial agent against a bacterial pathogen, the minimum inhibitory concentration (MIC) of the antimicrobial for the pathogen is usually determined through the agar dilution, broth dilution, or disc diffusion method [12-13].

The aim of this study is to determine the effectiveness of selected broad spectrum antibiotics, using agar dilution method, in restricting the growth of Pseudomonas species isolated through the use of agar media supplemented with Chloramphenicol.

\section{Material and methods}

\subsection{Isolation of chloramphenicol resistant Pseudomonas species from water}

Water samples were collected from a gutter within the Rivers State University campus, Nigeria, and from a river located near the University. The water samples were collected using $50 \mathrm{ml}$ sterile water sampling bottles. The samples were taken to the Microbiology laboratory of the Rivers State University, and analyzed for total heterotrophic bacterial count using nutrient agar (Titan Biotech Ltd., India), and greenish pigment producing Pseudomonas species using nutrient agar supplemented with $50 \mu \mathrm{g} \cdot \mathrm{ml}^{-1}$ chloramphenicol. Inoculated nutrient agar plates were incubated at $37{ }^{\circ} \mathrm{C}$ for 24 hours, while the inoculated nutrient agar plates supplemented with chloramphenicol were incubated at ambient temperatures $\left(28-32^{\circ} \mathrm{C}\right)$ for 48 hours.

\subsection{Identification of the isolates}

Greenish pigment producing colonies that grew on nutrient agar supplemented with $50 \mu \mathrm{g} . \mathrm{ml}^{-1} \mathrm{chloramphenicol} \mathrm{were}$ sub-cultured unto sterile nutrient agar plates to get pure isolates, and then stock culture of the pure isolates were prepared. The isolates were subjected to the Gram staining and microscopic examination, and the following physicochemical/biochemical tests: catalase, oxidase, motility, citrate utilization, indole production, MRVP (Methyl RedVogues Proskauer), blood haemolysis, casein hydrolysis, lecithinase production, and fermentation tests using glucose, lactose, maltose, sucrose, mannitol, xylose, starch, and glycerol.

\subsection{Preparation of selected chloramphenicol resistant Pseudomonas species for MIC determination}

Broth cultures of three of the isolates confirmed to belong to Pseudomonas species were prepared by inoculating the isolates into sterile Nutrient broth (Titan Biotech Ltd., India). The inoculated broths were incubated at $37{ }^{\circ} \mathrm{C}$ for 24 hours. After 24 hours, the absorbances of the broths were read using a 721 VIS Spectrophotometer (Huanghua Faithful Instrument Co. Ltd, China) set at $600 \mathrm{~nm}$. The turbidity of the broths was then adjusted, using sterile normal saline, to the turbidity of McFarland standard No. 1 which is agreed to portray a broth culture having a cell density of about $3 \times$ $10^{8} \mathrm{cfu} / \mathrm{ml}$ [14]. The absorbance of the McFarland standard at $600 \mathrm{~nm}$ as determined using the 721 VIS Spectrophotometer was 0.192 . The volume of normal saline added to the broth cultures to achieve the turbidity of McFarland standard was determined using some arithmetic calculations.

\subsection{Determination of the MIC of selected broad spectrum antibiotics against the selected isolates}

The MIC of three broad spectrum antibiotics (ciprofloxacin, erythromycin, and tetracycline) against the three isolates was determined using the agar dilution method [12]. Different sets of nutrient agar plates having increasing concentrations of each of the antibiotics were prepared. The concentrations of the antibiotics used include $0.625,1.25$, $2.5,5.0,10.0,20.0,40.0,80.0,160.0,320.0$, and $640.0 \mu \mathrm{g} / \mathrm{ml}$. To achieve these concentrations in the nutrient agar plates, stock solutions of $1000 \mu \mathrm{g} / \mathrm{ml}$ of the antibiotics were first prepared. The volume of the antibiotic stock solutions to be added to prepared molten agar medium so as to achieve the different concentrations were determined using equation 1 (Eq. 1), adapted from the equation $M_{1} V_{1}=M_{2} V_{2}$ [15]. 


$$
V_{A B}=\frac{\mathrm{M}_{\mathrm{AB}} \times \mathrm{V}_{\mathrm{MD}}}{1000 \mu \mathrm{g} / \mathrm{ml}}
$$

Where $\mathrm{V}_{\mathrm{AB}}$ is the volume of the antibiotic stock solution required, $\mathrm{M}_{\mathrm{AB}}$ is the antibiotic concentration specified for the nutrient agar medium, and $\mathrm{V}_{\mathrm{MD}}$ is the volume of the nutrient agar medium to be prepared.

On preparation of the nutrient agar media, after sterilization, the media were allowed to cool to about $50{ }^{\circ} \mathrm{C}$ before addition of the calculated volume of the antibiotic stock solution. The media now containing the antibiotics were poured into appropriately labeled sterile Petri dishes, allowed to solidify, and then dried in a size one hot box oven (Gallenkamp and Co. Ltd., England) set at $50^{\circ} \mathrm{C}$. Broth cultures $(0.1 \mathrm{ml})$ of the isolates adjusted to the McFarland No. 1 standard were inoculated onto their respective plates containing the antibiotics, and incubated at $37^{\circ} \mathrm{C}$ for 24 hours. After incubation, the plates were checked for the presence or absence of growth. The presence or absence of growth in each set of plates was then used to adjudge the MIC of the antibiotics against the isolates.

\section{Results}

\subsection{Bacterial populations of the water samples}

The total heterotrophic bacterial population (THB) and the greenish pigment producing bacterial population of water samples from the gutter and the river is presented in Table 1. From the Table it can be seen that the greenish pigment producing bacterial population of the samples are lower than the total heterotrophic bacterial population.

Table 1Bacterial population of the sampled water bodies

\begin{tabular}{lll}
\hline Sample & THB (cfu/ml) & GPB $(\mathbf{c f u} / \mathbf{m l})$ \\
\hline GT & $2.17 \times 10^{6}$ & 15 \\
RW & $2.02 \times 10^{5}$ & 1
\end{tabular}

THB - total heterotrophic bacterial population, GPB - greenish pigment producing bacterial population, GT - gutter water, RW - river water

\subsection{Identity of the greenish pigment producing bacterial isolates}

Table 2 Identification test results of selected greenish pigment producing bacterial isolates

\begin{tabular}{lllll}
\hline Test & CH1 & CH2 & CH3 & CH4 \\
\hline Gram staining reaction & - rods & - rods & - rods & - rods \\
Catalase & + & + & + & + \\
Oxidase & + & + & + & + \\
Motility & + & + & + & + \\
Citrate utilization & + & + & + & + \\
Indole & + & - & - & - \\
Vogues Proskauer & - & - & - & - \\
Methyl red & - & - & - & - \\
Haemolysis on blood agar & $\beta-H$ & $\beta-H$ & $\beta-H$ & $\beta-H$ \\
Casein hydrolysis & + & + & + & + \\
lecithinase production & + & + & + & + \\
Glucose fermentation & $\mathrm{A}$ & $\mathrm{A}$ & $\mathrm{A}$ & 0 \\
Lactose fermentation & 0 & 0 & 0 & 0 \\
Xylose fermentation & $\mathrm{A}$ & 0 & 0 & $\mathrm{~A}$ \\
Maltose fermentation & 0 & 0 & 0 & 0 \\
Mannitol fermentation & $\mathrm{A}$ & 0 & 0 & $\mathrm{~A}$ \\
Sucrose fermentation & 0 & 0 & 0 & 0 \\
Starch fermentation & $\mathrm{A}$ & 0 & 0 & 0 \\
Glycerol fermentation & $\mathrm{A}$ & $\mathrm{A}$ & 0 & 0 \\
\hline \multicolumn{2}{c}{$\beta$-H - beta haemolysis, A - only acid produced, $0-$ no change. }
\end{tabular}

$\beta-\mathrm{H}$ - beta haemolysis, $\mathrm{A}$ - only acid produced, 0 - no change. 
Selected greenish pigment producing the isolates were coded as follows; $\mathrm{CH} 1, \mathrm{CH} 2, \mathrm{CH} 3$, and CH4. Results generated from microscopic examination of their stained cells, and the physicochemical/biochemical tests carried out on them is presented in Table 2. On scrutiny of Table 2, it can be seen that the isolates displayed almost similar results for the different physicochemical/biochemical tests used.

\subsection{MIC of the broad spectrum antibiotics against the isolates}

Table 3MIC determination of ciprofloxacin against the isolates

\begin{tabular}{llcc}
\hline $\begin{array}{l}\text { Ciprofloxacin } \\
(\boldsymbol{\mu g} / \mathrm{ml})\end{array}$ & CH1 & CH3 & $\begin{array}{c}\text { CH4 } \\
\text { (presence }\end{array}$ \\
\hline 0.625 & + & absence of growth) \\
1.250 & + & + & + \\
2.500 & + & + & + \\
5.000 & - & + & + \\
10.00 & - & + & - \\
20.00 & - & + & - \\
40.00 & - & + & - \\
80.00 & - & + & - \\
160.0 & - & + & - \\
320.0 & - & + & - \\
640.0 & - & - & - \\
\hline
\end{tabular}

Determination of the MIC of the antibiotics, ciprofloxacin, erythromycin, and tetracycline, against the isolates CH1, CH3, and $\mathrm{CH} 4$ is presented in Table 3 - 5. From the Tables it can be seen that the MIC of ciprofloxacin against isolate $\mathrm{CH} 1$, $\mathrm{CH} 3$, and $\mathrm{CH} 4$ is $5 \mu \mathrm{g} / \mathrm{ml}, 640 \mu \mathrm{g} / \mathrm{ml}$, and $5 \mu \mathrm{g} / \mathrm{ml}$ respectively; the MIC of tetracycline against the isolates is $20 \mu \mathrm{g} / \mathrm{ml}$, $640 \mu \mathrm{g} / \mathrm{ml}$, and $5 \mu \mathrm{g} / \mathrm{ml}$ respectively; the MIC of erythromycin against the isolates is $40 \mu \mathrm{g} / \mathrm{ml}, 80 \mu \mathrm{g} / \mathrm{ml}$, and $80 \mu \mathrm{g} / \mathrm{ml}$ respectively. It can thus be deduced that isolate $\mathrm{CH} 3$ was resistant to ciprofloxacin and tetracycline, while isolate $\mathrm{CH} 1$ and $\mathrm{CH} 4$ were fairly susceptible to the three antibiotics.

Table 4 MIC determination of tetracycline against the isolates

\begin{tabular}{llcc}
\hline Tetracycline & CH1 & CH3 & CH4 \\
$(\mu \mathrm{g} / \mathrm{ml})$ & (presence & absence of growth) & ( \\
\hline 0.625 & + & + & + \\
1.250 & + & + & + \\
2.500 & + & + & + \\
5.000 & + & + & - \\
10.00 & + & + & - \\
20.00 & - & + & - \\
40.00 & - & + & - \\
80.00 & - & + & - \\
160.0 & - & + & - \\
320.0 & - & + & - \\
640.0 & - & - & - \\
\hline
\end{tabular}


Table 5 MIC determination of Erythromycin against the isolates

\begin{tabular}{llll}
\hline Erythromycin & CH1 & CH3 & CH4 \\
$(\mu \mathrm{g} / \mathrm{ml})$ & (presence $/$ & absence of & growth) \\
\hline 0.625 & + & + & + \\
1.250 & + & + & + \\
2.500 & + & + & + \\
5.000 & + & + & + \\
10.00 & + & + & + \\
20.00 & + & + & + \\
40.00 & - & + & + \\
80.00 & - & - & - \\
160.0 & - & - & - \\
320.0 & - & - & - \\
640.0 & - & - & - \\
\hline
\end{tabular}

\section{Discussion}

Culturing environmental or clinical samples on agar media supplemented with antibiotics for the isolation of greenish pigment producing Pseudomonas species can lead to the emergence of multidrug resistant Pseudomonas species. Infections caused by multidrug resistant species of Pseudomonas can be tackled through the use of broad spectrum antibiotics.

In this research, the effectiveness of three broad spectrum antibiotics (ciprofloxacin, tetracycline, and erythromycin) against Pseudomonas species isolated through the use of nutrient agar supplemented with $50 \mu \mathrm{g} / \mathrm{ml} \mathrm{chloramphenicol}$ was investigated. Colonies of Pseudomonas species that grew on the media were initially identified by the green pigment they produced which diffused into the media. The similar results displayed by the isolates are similar to the results usually displayed by species of Pseudomonas to the physicochemical/biochemical tests used. This conclusion was drawn by comparison with information in Prescott et al. [9], Lysenko [16], and Reynolds et al. [17].

The MIC of ciprofloxacin, tetracycline, and erythromycin against three of the green pigment producing Pseudomonas isolates were determined so as to know the effectiveness of the antibiotics in treating infections that may be caused by antibiotic-resistant Pseudomonas species. From the results obtained it can be deduced that one of the Pseudomonas isolate was highly resistant to ciprofloxacin and tetracycline; the MIC of these antibiotics against the isolate was as high as $640 \mu \mathrm{g} / \mathrm{ml}$. This is in agreement with the work of Hamud-Socoro [18], in which it was shown that a species of Pseudomonas $(P$. aeruginosa) became resistant to tetracycline after been grown in a medium containing low concentration of the antibiotic. In another related work, it was shown that a strain of $P$. aeruginosa has intrinsic resistance to tetracycline and some other antibiotics [19]. However, strains of another species of Pseudomonas (P. fluorescens) have been shown to be highly susceptible to tetracycline at a MIC of $0.305 \mu \mathrm{g} / \mathrm{ml}$ [20]. The strains were variants resistant to penicillin G, streptomycin, lincomycin and rifampicin which were developed from a colistinsensitive isolate of $P$. fluorescens LRC-R73. In this work one of the Pseudomonas isolate was susceptible to tetracycline at a MIC of $5 \mu \mathrm{g} / \mathrm{ml}$, and another at a MIC of $20 \mu \mathrm{g} / \mathrm{ml}$. Due to the differences in response to tetracycline, it can be concluded that the three Pseudomonas isolates belong to different species.

Two of the Pseudomonas isolates were susceptible to ciprofloxacin at a MIC of $5 \mu \mathrm{g} / \mathrm{ml}$, while one of the isolate was highly resistant to the antibiotic. A species of Pseudomonas ( $P$. aeruginosa) have been shown to become resistant to ciprofloxacin after exposure to the antibiotic [21]. However, only a small population of the Pseudomonas species survived treatment with the antibiotic, and became resistant to it. Pseudomonas species, especially P. aeruginosa, are usually fully susceptible to ciprofloxacin [22]. Resistance to ciprofloxacin occurs due to mutation in certain genes, and at a slow rate. Also only a small fraction of the population may become resistant after exposure. As seen in this study only one of the Pseudomonas isolates was resistant to ciprofloxacin. 
The three Pseudomonas isolates were susceptible to erythromycin at MIC values ranging from $40-80 \mu \mathrm{g} / \mathrm{ml}$. Not much work on the activity of erythromycin on Pseudomonas species can be found in online libraries. However, in a review carried out by Morita et al. [23], P. aeruginosa cells are said to be intrinsically resistant to macrolides, and that the MIC of erythromycin for a strain of $P$. aeruginosa is about $512 \mu \mathrm{g} / \mathrm{ml}$. The variance observed between the MIC in the review and this work could be attributed to the methods, media, quantity of cells used, and strain of the organisms investigated. For instance, in the review the MIC was cited for test carried out using Mueller-Hinton broth, whereas in this work the MIC was determined using an all purpose medium, and a solid medium not a liquid medium (broth).

The confirmation or disapproval of the effectiveness of erythromycin against resistant Pseudomonas species can be achieved by further research in this area. Also, future work can be carried out to determine the effectiveness of erythromycin against resistant isolates of other bacterial genus. However, for the time been, it has been shown in this work that erythromycin is effective against Pseudomonas species that are resistant to ciprofloxacin and tetracycline.

\section{Conclusion}

Multidrug resistant species of Pseudomonas can be generated in the laboratories as a result of using chloramphenicol for the isolation of Pseudomonas. Escape of these organisms from Microbiology laboratories can lead to the emergence and spread of infections that could be difficult to treat. More often, infections caused by multidrug resistant bacteria are treated with the use of broad spectrum antibiotics. It thus becomes important to know which broad spectrum antibiotic will actually work in such case scenario. In this work it has been shown that erythromycin is effective against Pseudomonas species that are resistant to ciprofloxacin and tetracycline as a result of growth on medium containing chloramphenicol. This implies that erythromycin could be used in the treatment of infections caused by multidrug resistant Pseudomonas species.

\section{Compliance with ethical standards}

\section{Disclosure of conflict of interest}

There are no conflicts of interest.

\section{References}

[1] Abouseoud M, Yataghene A, Amrane A and Maachi R. (2010). Production of a biosurfactant by Pseudomonas fluorescens - Solubilizing and wetting capacity. Chemical Engineering Transactions, 20, 291-296.

[2] Garbi C, Casasús L, Martinez-Álvarez R, Robla JI and Martín M. (2006). Biodegradation of oxadiazon by a soil isolated Pseudomonas fluorescens strain CG5: Implementation in an herbicide removal reactor and modelling. Water Research, 40(6), 1217-1223.

[3] Otenio M H, da Silva MTL, Marques MLO, Roseiro JC and Bidoia ED. (2005). Benzene, Toluene and Xylene biodegradation by Pseudomonas putida CCMI 852. Brazilian Journal of Microbiology, 36(3), 258-261.

[4] Peekate LP and Abu GO. (2017). Use of chloramphenicol in the differential enumeration of greenish pigment producing Pseudomonas. Basic Research Journal of Microbiology, 4(4), 33-41.

[5] Butler T. (2011). Treatment of typhoid fever in the 21 st century: promises and shortcomings. Clinical Microbiology and Infection, 17(7), 959-963.

[6] Dasgupta A. (2012). Advances in antibiotic measurement. Advances in Clinical Chemistry, 56, 75-104.

[7] Rose PW, Harnden A, Brueggemann AB, Perera R, Sheikh A, Crook D and Mant D. (2005). Chloramphenicol treatment for acute infective conjunctivitis in children in primary care: a randomised double-blind placebocontrolled trial. The Lancet, 366(9479), 37-43.

[8] Supiyaphun P, Kerekhanjanarong V, Koranasophonepun J and Sastarasadhit V. (2000). Comparison of ofloxacin otic solution with oral amoxycillin plus chloramphenicol ear drop in treatment of chronic suppurative otitis media with acute exacerbation. Journal of the Medical Association of Thailand, 83(1), 61-68.

[9] Prescott LM, Harley JP and Klein DA. (1999). Microbiology, fourth edition. The McGraw-Hill companies Inc., New York, 471, 475, 685-689. 
[10] Ory EM and Yow EM. (1963). The use and abuse of the broad spectrum antibiotics. Journal of the American Medical Association, 185(4), 273-284.

[11] Campoli-Richards DM, Monk JP, Price A, Benfield P, Todd PA and Ward A. (1988). Ciprofloxacin: A review of its antibacterial activity, pharmacokinetics properties and therapeutic use. Drugs, 35(4), 373-447.

[12] Balouiri M, Sadik M and Ibnsouda SK. (2016). Methods for in vitro evaluating antimicrobial activity: A review. Journal of Pharmaceutical Analysis, 6, 71-79.

[13] Jorgensen JH and Ferraro MJ. (2009). Antimicrobial susceptibility testing: A review of general principle and contemporary practices. Clinical Infectious Diseases, 49, 1749-1755.

[14] Clinical and Laboratory Standards Institute. (2012). Methods for dilution antimicrobial susceptibility tests for bacteria that grow aerobically; approved standard-9th ed. CLSI document M07-A9. Clinical and Laboratory Standards Institute, Wayne, PA

[15] Manilla PN, Ogali RE and Uzoukwu BA. (2001). Undergraduate chemistry: Fundamental principles. Timi Hyacinth Enterprises, Lagos, Nigeria, 55-56.

[16] Lysenko 0. (1961). Pseudomonas - An attempt at a general classification. Journal of General Microbiology, 25, 379-408.

[17] Reynolds MT, Falkiner FR, Hardy R and Keane CT. (1979). Differentiation of fluorescent Pseudomonas by their effect on milk agar. Journal of Medical Microbiology, 12, 379-382.

[18] Hamud-Socoro AA. (2004). Pseudomonas aeruginosa resistance to tetracycline and triclosan. Cantaurus, 12, 7-9.

[19] Li X-Z, Livermore DM and Nikaido H. (1994). Role of efflux pump(s) in intrinsic resistance of Pseudomonas aeruginosa: Resistance to tetracycline, chloramphenicol, and norfloxacin. Antimicrobial Agents and Chemotherapy, 38(8), 1732-1741.

[20] Naghmouchi K, Le LC, Baah J and Drider D. (2012). Antibiotic and antimicrobial peptide combinations: synergistic inhibition of Pseudomonas fluorescens and antibiotic-resistant variants. Research in Microbiology, 163(2), 101-108.

[21] Su H-C, Ramkissoon K, Doolittle J, Clark M, Khatun J, Secrest A, Giddings MC. (2010). The development of ciprofloxacin resistance in Pseudomonas aeruginosa involves multiple response stages and multiple proteins. Antimicrobial Agents and Chemotherapy, 54(11), 4626-4635.

[22] Lomholt JA and Kilian M. (2003). Ciprofloxacin susceptibility of Pseudomonas aeruginosa isolates from keratitis. British Journal of Ophthalmology, 87(10), 1238-1240.

[23] Morita Y, Tomida J and Kawamura Y. (2013). Responses of Pseudomonas aeruginosa to antimicrobials. Frontiers in Microbiology, 4, 1-8.

\section{How to cite this article}

Peekate LP and Biidee B. (2018). Effectiveness of selected broad spectrum antibiotics against Pseudomonas species isolated through the use of chloramphenicol. GSC Biological and Pharmaceutical Sciences, 5(3), 99-105. 